\title{
Los efectos de las políticas de calidad en las licenciaturas en Colombia: balance y alternativas
}

The Effects of Quality Policies on the B. A. in Colombia. Balance and Alternatives

Os efeitos das políticas de qualidade nos graus na Colômbia. Equilíbrio e alternativas

Sandra Patricia Rodríguez-Ávila* iD orcid.org/0000-0003-2687-135X

Olga Cecilia Díaz-Flórez** iD orcid.org/0000-0003-1002-9483

Diego Hernán Arias-Gómez *** iD orcid.org/0000-0002-0116-0896

Para citar este artículo: Rodríguez-Ávila, S., Díaz-Flórez, O. y Arias-Gómez, D. (2021). Los efectos de las políticas de calidad en las licenciaturas en Colombia: balance y alternativas. Revista Colombiana de Educación, l(81), 35-60. https://doi.org/10.17227/rce.num81-10688

\begin{tabular}{lr}
\hline (C) $\bigcup_{\mathbf{B Y}} \$ \mathbf{N C}$ & Recibido: $02 / 11 / 2019$ \\
Evaluado: $31 / 03 / 2020$
\end{tabular}

* Doctora en Historia. Profesora de la Universidad Pedagógica Nacional, Bogotá, Colombia

** Doctora en Educación. Profesora Universidad Pedagógica Nacional, Bogotá, Colombia Correo: odiaz@pedagogica.edu.co

*** Doctor en Educación. Profesor de la Universidad Distrital Francisco José de Caldas, Bogotá, Colombia. Correo: dhariasg@udistrital.edu.co 


\title{
Resumen
}

A partir de la indagación documental del Sistema Nacional de Acreditación (SNA), de los indicadores del Ministerio de Educación Nacional y de la información obtenida en varios foros regionales y nacionales con los responsables de los procesos de aseguramiento de la calidad en los programas de licenciatura, este artículo de investigación muestra la manera como se redujeron las licenciaturas y se afectó la autonomía universitaria entre el 2015 y el 2019, por efecto de la imposición de la acreditación obligatoria contemplada en la Ley 1753 de 2015, que aprobó el Plan Nacional de Desarrollo 2014-2018 "Todos por un Nuevo País". El análisis de estos impactos en los programas de formación de maestros se deriva de los referentes conceptuales que controvierten el enfoque del capital humano que ha orientado las políticas educativas y enfatiza en la construcción de lineamientos alternativos de calidad, desde los postulados de justicia social propuestos por Nancy Fraser (2008).

\section{Palabras clave}

formación de profesores; profesión docente; aseguramiento de la calidad; evaluación educativa; política educativa

\section{Keywords}

Teacher Training, Teaching

(Occupation), Quality Assurance,

Educational Assessment, Educational Policy

\begin{abstract}
This article is based upon a documentary search of the National Accreditation System, the indicators of the Ministry of National Education, and the information from different regional and national forums with the heads of quality assurance in the B. Ed. Programs. This article r shows how the Bachelors of Education were reduced, and university autonomy was affected between 2015 and 2019, due to the imposition of the mandatory accreditation contemplated in Ley 1753 of 2015, which approved the Plan Nacional de Desarrollo 2014-2018 [National Development Plan] "All for a New Country". The analysis of these impacts on teacher training programs arises from concept referents that question the human capital approach which has guided educational policies, and puts an emphasis on the creation of alternative quality guidelines, from the social justice postulates proposed by Nancy

Fraser (2008)
\end{abstract}

\begin{abstract}
Resumo
Com base na investigação documental do Sistema Nacional de Acreditação (SNA), dos indicadores do Ministério da Educação Nacional e das informações obtidas nos diversos fóruns regionais e nacionais com os responsáveis pelos processos de garantia da qualidade nos cursos de graduação, este artigo de pesquisa mostra como os graus de bacharelado foram reduzidos e a autonomia da universidade foi afetada entre 2015 e 2019, devido à imposição do credenciamento obrigatório previsto na Lei 1753 de 2015, que aprovou o Plano Nacional de Desenvolvimento 2014-2018 "Todos para um novo país". A análise desses impactos nos programas de formação de professores deriva dos referenciais conceituais que contestam a abordagem do capital humano que tem norteado as políticas educacionais e enfatizam a construção de diretrizes alternativas de qualidade, a partir dos postulados de justiça social propostos por Nancy Fraser (2008).
\end{abstract}

\section{Palavras-chave}

formação de professores; qualidade da educação; profissão docente; política de educação; desigualdade social 


\section{Introducción}

En el 2014 el Ministerio de Educación Nacional (MEN) suscribió el Convenio 707 con el Departamento Administrativo de Ciencia Tecnología e Innovación (Colciencias), para fomentar la investigación en Educación Superior mediante la financiación de propuestas que estudiaran "mecanismos para mejorar la calidad de la educación superior en lo referente a los programas de licenciaturas", con el fin de "conocer cuáles son los avances y el impacto de la acreditación sobre los programas de licenciatura y sus posibilidades reales de avanzar hacia un sistema en el que la alta calidad pueda ser alcanzada por la mayoría de estos programas".

A partir de estos lineamientos, Colciencias (actual Minciencias) abrió una invitación a distintas instituciones de educación superior (IES) para presentar proyectos que reconocieran y analizaran las brechas que afectaban la calidad de los programas de licenciatura en Colombia y que, con base en sus resultados, diseñaran estrategias para disminuir estas brechas y lograr así la acreditación de estos programas. ${ }^{1}$

La propuesta de la Universidad Pedagógica Nacional fue seleccionada en el marco de dicha invitación y los resultados de este trabajo mostraron, ${ }^{2}$ contrario a lo sostenido por otros estudios, que el problema central radica en el marco político desde el cual se vienen formulando las propuestas de formación de profesores, ${ }^{3}$ basadas en las teorías del capital humano. Dichos planteamientos asumen la desigualdad como brechas de calidad y no como una situación derivada de la inequitativa distribución de recursos, de la falta de reconocimiento y de la ausencia de canales efectivos de participación en las decisiones políticas que afectan, en este caso, a los programas de licenciatura y a sus comunidades académicas.

1 En el documento de convocatoria se definieron las brechas de calidad a partir de los lineamientos del Consejo Nacional de Acreditación (CNA), en lo referido a: capacidades en investigación y producción intelectual, desarrollo curricular, formación y desarrollo profesoral, y resultados de pruebas estandarizadas (Saber Pro).

2 Esta investigación fue realizada por la Universidad Pedagógica Nacional entre los años 2016 y 2017 y sus resultados fueron publicados en el 2018 en el libro titulado Entre las exigencias de calidad y las condiciones de desigualdad: formación inicial de profesores en Colombia. (Arias et ál, 2008).

3 Se analizaron los documentos de organismos multilaterales (Unesco, Oreal/Unesco, Banco Mundial, y la OCDE) y los planes nacionales de Desarrollo y sectoriales de Educación (Arias et ál., 2018). 
A partir de los mismos sistemas de información desde los cuales se ha concluido que se debe fortalecer la calidad de las licenciaturas mediante su perentoria acreditación, ${ }^{4}$ esta investigación mostró otros problemas que efectivamente afectan la calidad de las licenciaturas más que la obtención de un reconocimiento, que ignora los contextos locales y regionales de estos programas, en donde se expresa con mayor rigor la desigualdad que se busca soslayar con la nominación de brechas.

Aunque esta investigación fue cofinanciada con recursos del MEN, las políticas de calidad de las licenciaturas que se pusieron en marcha entre el 2015 y el 2019 siguieron las orientaciones de otro tipo de estudios proclives a las formulaciones de la política internacional. En este artículo nos proponemos mostrar los efectos de esta política derivada de estudios que no consultan la realidad nacional en materia educativa y formulamos una propuesta de lineamientos de calidad derivados de la investigación mencionada, que contempló la participación de las comunidades académicas que se encargan de la formación de profesores en los programas de licenciatura en distintas regiones del país. Finalmente, hacemos algunas reflexiones desde los postulados de la justicia social sobre la manera como la política que ha orientado la calidad ha incentivado la desigualdad en Colombia, que se encuentra como lo anotan Urrutia y Robles, "dentro de los países más desiguales de la región más desigual del mundo" (2019, p. 75).

\section{Los efectos de la acreditación obligatoria de las licenciaturas en Colombia}

En el marco de la Constitución Política de Colombia, cada cuatro años se debe proyectar un Plan Nacional de Desarrollo en el cual se formulan los lineamientos de las políticas públicas con las cuales el poder ejecutivo administra el Estado en cada periodo presidencial. ${ }^{5}$ Este documento, además de establecer los objetivos del Gobierno, sirve de instrumento para la evaluación de la gestión pública. Según la Constitución Política de 1991, los planes de desarrollo se componen de dos grandes apartados: uno general, en el cual se formulan los propósitos nacionales a largo plazo y las estrategias que orientan la acción del Estado en materia de

4 Se analizó la información del Sistema de Aseguramiento de la Calidad de la Educación Superior en Colombia (Sistema Nacional de Información de la Educación Superior-SNIES, Sistema para la Prevención de la Deserción en las Instituciones de Educación Superior-SPADIES-, Observatorio Laboral de Educación -OLE-y Sistema de Información para el Aseguramiento de la Calidad-SACES-) y los datos de los resultados de las pruebas de competencias genéricas del examen Saber Pro (Arias et ál., 2018).

5 Los planes de desarrollo están regulados por la Ley orgánica del Plan de Desarrollo (Ley 152 de 1994), que incluye los principios básicos, las autoridades y los procedimientos para la formulación, aprobación, ejecución y evaluación de los planes de desarrollo, bajo la orientación del Departamento Nacional de Planeación. 
política económica, social y ambiental; y el otro, con la formulación de los principales programas de inversión del Gobierno, con énfasis en los recursos y las fuentes de financiación.

Los planes de desarrollo tienen la misma vigencia de los periodos presidenciales, lo cual permite inferir que las transformaciones que inciden en las normas estatutarias, como la Ley 30 de 1992 que organiza el servicio público de la Educación Superior, deberían cursar un proceso diferente; sin embargo, en el Plan de Desarrollo del último Gobierno de Juan Manuel Santos (2014-2018), se incorporó un cambio sustancial referido a la calidad de los programas académicos que forman profesionales de la educación, lo cual generó un esfuerzo adicional de las comunidades académicas comprometidas con este tipo de formación, para mantener vigentes las licenciaturas.

En efecto, con la expedición de la Ley 1753 del 2015, que aprobó el Plan Nacional de Desarrollo 2014-2018 Todos por un Nuevo País (Departamento Nacional de Planeación-DNP, 2015), la acreditación de alta calidad establecida como un proceso voluntario en la Ley 30 de 1992 se convirtió en un requisito obligatorio de manera exclusiva para los programas de licenciatura. Se fijaron además unos plazos perentorios de cumplimiento que implicaban, por un lado, nuevos requerimientos de calidad para los programas que se encontraban en procesos de autoevaluación para obtener la renovación de registros calificados o de la acreditación de calidad y, por otro, el cierre de los programas que no lograran alcanzar este nuevo requisito obligatorio en el plazo establecido por esta norma nacional. ${ }^{6}$

Con la promulgación de la Ley 1955 del 2019 que aprobó el Plan Nacional de Desarrollo 2018-2022 Pacto por Colombia, Pacto por la Equidad (DNP, 2015), quedó sin vigencia la obligatoriedad de la acreditación para las licenciaturas. No obstante, los efectos de la Ley 1753 del 2015 en estos programas académicos no pueden soslayarse porque, como se mostrará a continuación, durante los cuatro años en los que estuvo vigente, tuvo implicaciones significativas para los programas de licenciatura y para las comunidades académicas que los respaldan.

Las Bases del Plan Nacional de Desarrollo 2014-2018: Todos por un Nuevo País, que sustentan la Ley 1753 del 2015, se fundamentaron en el informe de la Fundación Compartir Tras la excelencia docente. Cómo mejorar la calidad de la educación para todos los colombianos (García et ál., 2014). Desde sus planteamientos, el Gobierno nacional se propuso convertir

6 La Ley 1753 del 2015 estableció que los programas de licenciatura con mínimo cuatro cohortes de egresados debían obtener acreditación de alta calidad en dos años, de lo contrario perderían la vigencia del registro calificado que les permite funcionar. Se propuso además nivelar los criterios de calidad de los requisitos exigidos para el registro calificado con los que se exigían para la acreditación de calidad mediante nuevas disposiciones normativas y técnicas que el MEN debía formular en los mismos dos años de plazo que se otorgó a las licenciaturas para obtener la acreditación. 
la educación en un instrumento para la igualdad social, bajo el supuesto de que mediante su promoción se nivelan "las oportunidades de las personas" y se abren "puertas de progreso y mejora la calidad de la democracia" (Arias et ál., 2018, p. 90), lo cual podría "convertir en el año 2025 a Colombia en la más educada" (DNP, 2015, p. 77). ${ }^{7}$ Para lograr este objetivo se propuso cerrar las brechas de calidad y pertinencia que, según los resultados de las pruebas censales y nacionales y los indicadores del MEN y del CNA, podrían depender de la calidad docente (formación y estabilidad laboral).

Según el Gobierno nacional, para cerrar las brechas en acceso y calidad era necesario incentivar la formación del capital humano, incrementar la movilidad social y fomentar la construcción de ciudadanía mediante las siguientes líneas de acción: potenciar la educación inicial; alcanzar la calidad educativa en educación básica y media; incrementar la cobertura y la permanencia en el sistema educativo; construir el sistema de educación terciaria; avanzar con el Sistema Educativo Indígena Propio (SEIP) en el marco del Decreto 1953 del 2014; reducir a cero el analfabetismo; promover el bilingüismo y crear las condiciones para potenciar las capacidades y los talentos excepcionales (DNP, 2015).

En el marco del Sistema de Formación de Capital Humano, la segunda línea de acción antes mencionada, apuntó al aseguramiento de la calidad, entendida como la garantía de que los procesos de formación satisfagan condiciones o estándares mínimos en instituciones y programas, que conduzcan al desarrollo efectivo de las competencias y aprendizajes de los estudiantes. Mediante el rediseño de los criterios para otorgar registros calificados de los programas de licenciatura, se buscó promover la acreditación de alta calidad de estos programas, fomentar la formación avanzada del cuerpo docente, aumentar la eficiencia del Sistema de Aseguramiento de la Calidad en sus funciones de evaluación, inspección, vigilancia y control, incorporar la Formación para el Trabajo y Desarrollo Humano en el modelo de calidad y estandarizar los criterios de calidad del Consejo Nacional de Acreditación (CNA) y de la Comisión Nacional Intersectorial de Aseguramiento de la Calidad de la Educación Superior (Conaces) para la expedición de registros calificados y propiciar la autogestión y rendición de cuentas de las IES (mecanismos preventivos y correctivos).

La pretensión de la formación de capital humano que orienta este PND (DNP, 2015) se basa en postulados que por el mismo periodo formuló el Banco Mundial (Bruns y Luque, 2014), en torno a la ahora denominada "excelencia docente", según la cual la calidad de los maestros es uno de

7 Eslogan utilizado para promover las políticas del Gobierno en dicho periodo y que forma parte de los ejes del PND 2014-2018 (tercer eje: Colombia, la más educada). 
los determinantes centrales del aprendizaje. Para alcanzarla, se "buscará atraer, formar y promover al mejor capital humano para que sean los docentes del Sistema Educativo Colombiano" (DNP, 2015, p. 360).

En lo que respecta a la formación de profesores, las bases del PND se proponían promover la excelencia docente para mejorar el aprendizaje de los estudiantes, a partir del fomento de los programas de licenciatura con estándares altos de calidad y con requisitos de ingreso igualmente elevados para sus aspirantes. Se buscó que los mejores egresados de licenciaturas y carreras profesionales ingresaran a la carrera docente, para que se formaran durante el ejercicio profesional con créditos condonables; ${ }^{8}$ también se propuso evaluar su desempeño en función del mejoramiento continuo y mejorar los salarios para nivelar a los docentes con respecto a los ingresos de otros profesionales. ${ }^{9}$

En torno a las proyecciones allí señaladas, Ilama la atención el tipo de metas establecidas, y aquellas a las cuales finalmente no se destinaron los recursos requeridos para su concreción, que precisamente son las recomendaciones que la literatura ${ }^{10}$ ha señalado que tienen una importante repercusión en el estatus social de la profesión docente y en lograr atraer a los mejores bachilleres del país a la profesión, según se ilustra con las experiencias de otras regiones ${ }^{11}$ en los que "los estudiantes de programas de pedagogía en estos países reciben becas y subsidios de manutención durante sus estudios [...] y la remuneración es alta y competitiva al inicio y durante su carrera profesional" (García et ál., 2014, p. 21).

Por su parte, en cuanto a la meta de lograr que el porcentaje de programas de licenciatura con acreditación de alta calidad pasara del 11,4\% (línea base del 2013) al 15 \% (meta al 2018), contrasta con la exigencia de la obligatoriedad de este proceso, pues resulta paradójico que por una parte se presionó con la Ley 1753 para que los programas que cumplían los requisitos se acreditaran y sin embargo, la meta proyectada es baja si

8 La meta era pasar de un 28,5 \% (línea base del 2013) de docentes oficiales de educación preescolar, básica y media con formación de posgrado a un 68 \% (meta al 2018). Asimismo, se planteó pasar de una línea base (2013) de 38 docentes o aspirantes docentes beneficiados con recursos de la nación para mejorar su formación al nivel de posgrado a 17000 (meta al 2018). (PND, 2015, p. 361).

9 Sobre este mecanismo no se formuló ninguna meta, por tanto no se destinaron recursos para lograr su concreción.

10 Nos referimos al informe Tras la excelencia docente presentado por García et ál. (2014), único estudio de referencia para sustentar estas reformas incluidas en el PND 2014-2018.

11 El estudio remite al análisis del marco de referencia internacional a partir de cuatro casos: Singapur, Finlandia, Canadá (Ontario) y Corea del Sur, los cuales son analizados en seis dimensiones: 1) formación previa al servicio, 2) selección, 3) retención y promoción, 4) evaluación para el mejoramiento continuo, 5) formación en servicio y 6) remuneración; las mismas dimensiones que incorporó el PND. 
se tiene en cuenta que la pérdida de la acreditación implicaba, según la misma ley, la pérdida del registro calificado. Al respecto cabe preguntarse ¿aspiraba entonces el Gobierno nacional disminuir expresamente el número de licenciaturas con la acreditación obligatoria, o simplemente los análisis técnicos en los que se basó el MEN no tuvieron en cuenta el efecto que podría generar su propia medida?

Los datos de la investigación antes citada (Arias et ál., 2018) pueden ayudar a responder estas preguntas. Para el 2016, el $17 \%$ de los programas de licenciatura (89) tenían la acreditación de alta calidad y el restante $83 \%$ (412 programas) contaban con registro calificado; si mantenemos estos datos como referencia y se tiene en cuenta que se buscaba incrementar la meta en un 3,6\%, eso significa que solo se esperaba que se mantuvieran 104 programas de licenciatura vigentes; una tendencia arbitraria además de generadora de nuevas y profundas desigualdades.

De hecho, a través de la expedición del Decreto 892 del 28 de mayo del $2017^{12}$ se intentó corregir parcialmente las nuevas problemáticas que surgieron, pues en esta norma se reconoce que para avanzar en la implementación de los planes especiales de educación rural (PDET) se debía evitar que los programas de licenciatura perdieran su registro calificado. Por esta razón, se amplió el plazo de acreditación a 32 meses a partir de mayo de 2017, para que los programas que se ofrecen en departamentos con municipios priorizados para la implementación de los PDET obtuvieran su acreditación. Al contrastar la información que sirvió de base para la investigación realizada por la Universidad Pedagógica Nacional (con corte a junio del 2016) y la que se utilizó para promulgar este decreto, se evidencia la falta de planeación del artículo 222 de la Ley 1753 del 2015 (tabla 1).

12 Este decreto incluye un amplio conjunto de considerandos clasificados en generales (referidos a la paz como derecho constitucional en el marco del punto uno del Acuerdo Final con las FARC-EP, correspondiente a la reforma rural integral, y en especial el numeral 1.3.2.2); formales y materiales, ambos de validez constitucional, que remiten al compromiso de incluir la disponibilidad y permanencia del personal docente calificado en las zonas donde se busca llevar a cabo el Plan Especial de Educación Rural. 
Tabla 1.

Estado de los programas de licenciatura entre junio del 2016 y mayo del 2017

\begin{tabular}{|c|c|c|c|c|}
\hline \multirow{2}{*}{$\begin{array}{c}\text { Estado de los } \\
\text { programas }\end{array}$} & \multicolumn{2}{|c|}{$\begin{array}{l}\text { Licenciaturas en } \\
\text { junio del } 2016^{<?>}\end{array}$} & \multicolumn{2}{|c|}{$\begin{array}{c}\text { Licenciaturas en } \\
\text { mayo del } 2017\end{array}$} \\
\hline & $\begin{array}{c}\text { Total } \\
\text { nacional }\end{array}$ & $\begin{array}{c}\text { Total } \\
\text { departamentos con } \\
\text { municipios } \mathrm{PDET}^{<?>}\end{array}$ & $\begin{array}{c}\text { Total } \\
\text { nacional }\end{array}$ & $\begin{array}{c}\text { Total } \\
\text { departamentos con } \\
\text { municipios } \text { PDET }^{<>>}\end{array}$ \\
\hline Programas activos & 501 & 277 & 352 & 244 \\
\hline Programas con acreditación & 89 & 33 & 89 & 33 \\
\hline Programas sin acreditación & 412 & 244 & 263 & 211 \\
\hline $\begin{array}{l}\text { Programas sin acreditación } \\
\text { que iniciaron proceso ante } \\
\text { el cNA }\end{array}$ & - & - & 175 & 99 \\
\hline $\begin{array}{l}\text { Programas sin acreditación } \\
\text { que no iniciaron proceso } \\
\text { ante el CNA }\end{array}$ & - & - & 88 & 55 \\
\hline $\begin{array}{l}\text { Programas que al } 9 \text { de } \\
\text { mayo del } 2017 \text { ( } 91,4 \% \\
\text { evaluado) cumplían el } \\
\text { requisito de acreditación } \\
\text { de calidad }\end{array}$ & - & - & - & 51 \\
\hline
\end{tabular}

Fuente: elaboración propia a partir de los datos de Arias, Díaz, Garzón, León, Rodríguez y Valbuena (2018), fecha de corte: 30 de junio del 2016, y de la consulta en snies (13 de agosto del 2019).

En el momento de formular el Decreto 892 del 2017, se planteó que existían 9200 estudiantes procedentes de los municipios priorizados para la implementación de los PDET y que el cierre de las licenciaturas podría afectar de manera significativa su ingreso a la educación superior porque, según el Observatorio Laboral, más de 1600 jóvenes recién egresados de los programas de licenciatura ingresan al sistema educativo, lo cual también afectaría las oportunidades laborales de las nuevas generaciones de maestros de estas zonas. Los cupos nuevos que se dejarían de ofertar cada año en los programas de licenciatura de las instituciones ubicadas en los departamentos con municipios priorizados para la implementación de lOS PDET ascendía en ese momento a 13250 y también se afectarían los convenios de las 13 escuelas normales superiores ubicadas en estas regiones, las cuales mediante el proceso de reconocimiento de saberes contemplado en el Decreto 1075 del 2015 lograban que sus estudiantes accedieran a la educación superior.

Además de estos efectos previstos por el mismo Gobierno nacional en el Decreto 892 del 2017, en el balance cuantitativo referido al modo en que se ha afectado la oferta de formación de maestros, los datos comparados entre junio 30 del 2016 y el 13 de septiembre del 2019), ilustran 
los problemas que provocaron estas medidas cinco años después de su promulgación. Según se aprecia en la tabla 2, el número total de programas de licenciatura disminuyó tanto en las instituciones del sector oficial como en las del privado: en el primer caso, en un $22 \%$ (65) y en el segundo en un $9 \%(17)$, pero fueron las del sector oficial las más afectadas. En el total, la diferencia fue de un $16 \%$ de programas, es decir, 82 menos respecto al 2016.

\section{Tabla 2.}

Comparación número total de programas de licenciaturas por sector, 2016 y 2019

\begin{tabular}{ccccc}
\hline \multirow{2}{*}{ Sector } & \multicolumn{4}{c}{ Total general } \\
\cline { 2 - 5 } & $\mathbf{2 0 1 6}$ & $\mathbf{2 0 1 9}$ & $\begin{array}{c}\text { Diferencia } \\
\mathbf{2 0 1 6 - 2 0 1 9}\end{array}$ & $\%$ \\
\hline Oficial & 300 & 235 & -65 & -22 \\
\hline Privada & 201 & 184 & -17 & -9 \\
\hline Total & 501 & 419 & -82 & -16 \\
\hline
\end{tabular}

Fuente: Elaboración propia a partir de los datos de Arias, et ál. (2018), fecha de corte: 30 de junio del 2016, y de la consulta en sNiEs (13 de agosto del 2019).

En relación con su condición frente al sistema de aseguramiento de la calidad, tal como se aprecia en la gráfica 1, la tendencia muestra que el mayor porcentaje de crecimiento de programas con acreditación se produjo en el sector oficial con 87 nuevos programas con este reconocimiento, mientras que en el privado fue de 35 programas, para un total de 122 .

Esta tendencia es consistente con la disminución en el número y porcentaje de programas que cuentan solo con el registro calificado y que corresponden a las licenciaturas que no cumplían con el requisito de las cuatro cohortes en el momento en que se expidió y operó la normatividad, o son programas nuevos posteriores a esta regulación. Para el caso del sector oficial, se disminuyó en 152 programas (65\%), mientras que para el privado fue de 52 programas $(29 \%)$; en total, la disminución fue del $50 \%$ (204), y se redujo de un $82 \%$ del total de programas con solo registro calificado a un $50 \%$ (gráfica 2 ). 


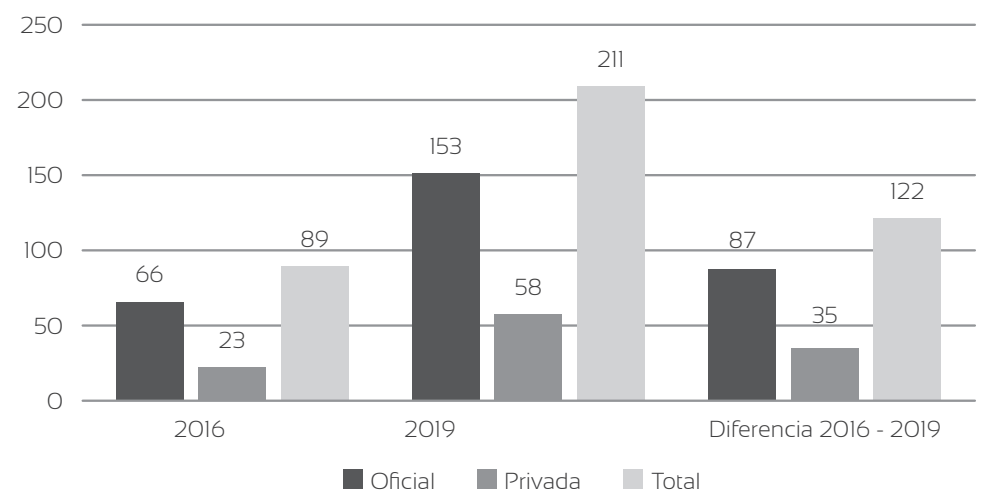

Gráfica 1. Comparación número de licenciaturas con acreditación por sector y total en el 2016 y el 2019.

Fuente: Elaboración propia a partir de los datos de Arias, et ál. (2018), fecha de corte: 30 de junio del 2016, y de la consulta en sNies (13 de agosto del 2019)

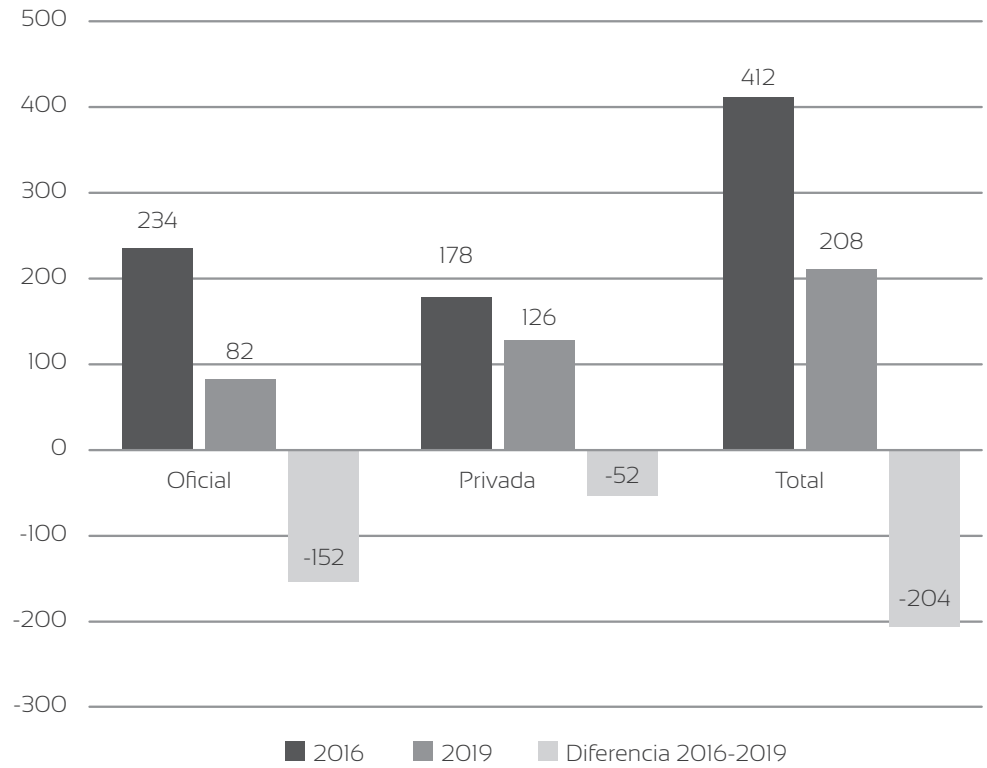

Gráfica 2. Comparación número de licenciaturas solo con registro calificado por sector en el 2016 y el 2019.

Fuente: Elaboración propia a partir de los datos de Arias, et ál. (2018), fecha de corte: 30 de junio del 2016, y de la consulta en sNIEs (13 de agosto del 2019).

Resulta cuestionable que los supuestos "estudios técnicos" que se reiteran en los considerandos de este decreto no hubieran contemplado la situación de las licenciaturas en las regiones, para optar por plantear como criterio unívoco de calidad, el volcar los esfuerzos institucionales en presionar la obtención de la acreditación, a partir del cumplimiento de 
requisitos imposibles de alcanzar en las condiciones de desigualdad en las cuales estos programas deben llevar a cabo los procesos de formación docente. No solo se afectó una norma estatutaria (Ley 30 de 1992) con una ley transitoria (Ley 1753 del 2015), sino que también se afectó la autonomía universitaria y se improvisó una medida que luego se intentó reparar parcialmente con el Decreto 892 del 2017.

\section{Los cambios normativos y sus implicaciones en la autonomía universitaria}

En sus casi cuatro años de vigencia, la Ley 1753 del 2015 incidió de manera decisiva en la formación previa al servicio (escuelas normales superiores y programas de licenciatura), transformó la naturaleza de la acreditación de calidad, considerada como un proceso voluntario en la Ley 30 de 1992 (artículo 53) y vulneró la autonomía de las IES, al condicionar a las licenciaturas a nuevos requisitos para mantener la vigencia de los programas, expresados en un conjunto normativo que exigió a los profesores universitarios responsables de ellos replantear permanentemente las agendas de trabajo de los equipos encargados del registro calificado y la acreditación de las licenciaturas, para cumplir con los propósitos que las bases del Plan Nacional de Desarrollo 2014-2018 establecieron como objetivos del cuatrienio.

Durante el segundo periodo presidencial de Juan Manuel Santos (20142018) se expidieron en total cuatro decretos, dos resoluciones y cuatro circulares entre el 2015 y el 2019, que ajustaron los requerimientos de calidad de las licenciaturas para los procesos de registro calificado y acreditación (Decreto 2450 del 2015, Resolución 2041 del 2016, y Resolución 18583 del 2017), que regularon tiempos y procedimientos (Circular 61 del 2015, Circular 14 del 2016 y Circular 16 del 2017), y que además plantearon modificaciones al Sistema de Aseguramiento de la Calidad (Decreto 1280 del 2018, Decreto 2389 del 2018, Decreto 1330 del 2019 y Circular 18 del 2018).

En el momento de la promulgación de varias de estas normas se establecieron requisitos de imposible cumplimiento inmediato, como los siguientes: limitar las denominaciones de los programas de licenciatura a aquellas que se corresponden con las áreas obligatorias y fundamentales del conocimiento (Ley 115 de 1994), o al grupo etario o poblacional al cual va dirigido el proceso formativo; ${ }^{13}$ definir contenidos curriculares y competencias del educador, lo que implicaba una reforma de los programas académicos, en contravía de lo establecido en los preceptos

13 Esto implicó delimitar a 52 las denominaciones de los programas de licenciatura (artículo 2, numeral 1). 
de la Constitución y la Ley 30 sobre la autonomía universitaria; realizar investigaciones disciplinares y pedagógicas articuladas a la comunidad académica internacional, lo que implicaba una mayor disponibilidad de recursos de inversión que en las IES públicas son insuficientes; contar con personal docente altamente calificado, con trayectoria en investigación y producción académica, con manejo de una segunda lengua y con experiencia de aula en los niveles de educación inicial, preescolar, básica o media, lo cual implicaba reorientar los perfiles de vinculación de los profesores universitarios; garantizar que los graduados de los programas de licenciatura contaran con un nivel de inglés A2 o superior ${ }^{14}$ del Marco Común Europeo de Referencia (MCER); y finalmente, establecer un mínimo de créditos presenciales de la práctica pedagógica ${ }^{15}$ y demostrar la existencia de convenios con instituciones educativas para su desarrollo.

Estos dos últimos requisitos obligaron a los programas a realizar varias transformaciones en un plazo inmediato, sin que estuvieran sustentados en ningún estudio o diagnóstico que permitiera valorar su pertinencia y sin formular mecanismos progresivos para que se pudieran llevar a cabo, pues todas estas exigencias eran de obligatorio cumplimiento en un plazo que no excedía el mes de junio del 2017 para los programas que no contaban con acreditación en el momento de expedición de la Ley 1753 del 2015. Por ejemplo, para el caso de la formación en lenguas extranjeras, no se contempló que:

Una lengua no se aprende por decreto: en el contexto colombiano, el proceso de aprendizaje de una lengua extranjera es atravesado por diferentes factores como las profundas brechas del desarrollo regional, el nivel socioeconómico, el capital cultural familiar, las posibilidades de inmersión y otras condiciones que facilitan o retrasan el aprendizaje de una segunda lengua en el nivel esperado. (Atehortúa, 2017).

Para el caso de la práctica pedagógica, además de obligar a reformar los programas en relación con los créditos académicos destinados a esta actividad, se definieron las condiciones en las cuales las IES debían realizar los convenios para su desarrollo. Al respecto el MEN expidió la Directiva 30 del 2016 en la cual extiende una invitación a los gobernadores, alcaldes y secretarios de Educación de entidades territoriales certificadas (ETC), a celebrar convenios entre estas ETC y las IES, para que los estudiantes de los programas de licenciatura pudiesen desarrollar su práctica pedagógica en establecimientos oficiales.

14 Nivel C1 en inglés, y nivel B1 o superior después de los tres primeros años de entrada en vigencia del Decreto 1075 del 2015.

15 Inicialmente fueron 50 créditos en la Resolución 2041 del 2016 y luego 40 en la Resolución 18583 del 2017. Es importante tener en cuenta que esta exigencia representaba, en el primer caso, un $31 \%$ aproximado de un plan de estudios de 160 créditos. 
Esta directiva no se envió a las IES que ofrecen licenciaturas, sino a las entidades territoriales, a las cuales además se les informó acerca de los alcances y propósitos de la Ley 1753 del 2015, con respecto a "los índices de calidad del servicio educativo en los niveles de preescolar, básica y media" para los cuales, una de las estrategias formuladas para el mejoramiento, es "la formación académica de quienes en el futuro aspiran a desempeñarse como docentes en instituciones educativas" (Directiva 30 del 2016). Según lo planteado por esta directiva, las prácticas pedagógicas, tal como se contemplan en el conjunto normativo emitido por el Ministerio:

[...] deben estar contempladas en los planes de estudio de los programas de licenciatura, dado que se constituyen en espacios formativos en contextos reales, que les permiten a los futuros maestros desarrollar las competencias para aprehender y apropiar el contenido disciplinar desde la perspectiva de enseñarlo y como objeto de enseñanza; saber cómo utilizar estrategias y prácticas que permitan resolver dificultades; y conocer cómo evaluar los aprendizajes desarrollados. (MEN, 2016).

Bajo el supuesto de que este tipo de alianzas inciden en la calidad educativa del país "a través de la formación de docentes con un conocimiento real de su actividad profesional", los estudiantes de las licenciaturas podrán Ilevar a cabo su práctica en el marco de lo establecido por las IES y por las orientaciones del acuerdo de voluntades provenientes de las ETC, a partir de los aspectos que el Ministerio considera relevantes. ${ }^{16}$

Esta directiva prescribe exhaustivamente los términos en los cuales deben celebrarse este tipo de convenios, ${ }^{17}$ condiciona el alcance mismo de asuntos referidos a la práctica pedagógica, exige acoger lo estipulado en la normatividad ministerial, propone negociar con las ETC los términos de

16 Según esta directiva, los aspectos sobre los cuales los estudiantes deben realizar su práctica son: 1) las dinámicas de aula y de la institución según contextos, 2) las modalidades de formación presencial y a distancia, 3) la relación dialógica entre la formación pedagógica y las disciplinas, 4) los componentes de los contenidos cuticulares y las competencias en las que se están formando como profesionales de la educación, y 5) sus propias prácticas pedagógicas.

17 Las ETC pueden realizar una convocatoria pública en sus regiones como lo establece el reglamento operativo del Comité Territorial de Capacitación (стс), la cual debe incluir la manifestación de intención de las IES, la distribución de establecimientos educativos por IES, la distribución del número de estudiantes de licenciatura por establecimiento educativo y por semestre, cronogramas de perfeccionamiento del convenio y de desarrollo de la práctica pedagógica y definición de responsabilidades de las partes. Los convenios deben suscribirse de conformidad con los principios definidos en el artículo 209 de la Constitución Política como acuerdos de voluntades según lo establece la Ley 80 de 1993. Siguiendo este marco jurídico los convenios deben precisar su modalidad (marco o específico); las partes involucradas (IES y ETC) y su competencia; el objeto referido a la dinamización de las prácticas pedagógicas; los compromisos, roles y responsabilidades de las partes; las condiciones para el desarrollo de las prácticas pedagógicas; los aspectos referidos a plazos, operación, procedimientos y coordinación del convenio; y la responsabilidad y cubrimiento de los practicantes al Sistema de Riesgos Laborales según el Decreto 1072 del 2015. 
los convenios y presentar los modelos de práctica, a partir de seis aspectos definidos en la misma Directiva. ${ }^{18}$ Además de estas prescripciones, se expresa taxativamente que no se contempla la inversión de recursos por parte de las ETC, lo cual puede tener efectos presupuestales para las IES, con respecto a la afiliación y el pago de los aportes al Sistema General de Riesgos Laborales que establece el Decreto 1072 del 2015.

Aunque en todos estos documentos se declara que las regulaciones se proponen en el marco de la autonomía universitaria, la definición de criterios curriculares, créditos académicos, mecanismos de evaluación, procesos de investigación e incluso la conceptualización de práctica docente y sus modalidades, finalmente tiene un efecto prescriptivo, que circunscribe y restringe la posibilidad efectiva de la autonomía universitaria, lo cual condiciona las dinámicas de las comunidades académicas que se encargan de los procesos de registro calificado y acreditación de calidad y sobre todo, restringen las posibilidades de diseñar proyectos de formación de maestros que consulten las particularidades y necesidades, especialmente de los contextos regionales.

\section{Alternativa política para formación inicial de maestros}

Contrario a esta ruta de presiones y exigencias que no posibilitan configurar alternativas para disminuir las desigualdades en las condiciones en que se desarrollan los procesos de formación inicial de los educadores que reclama este país, el equipo de investigación del proyecto aprobado por Colciencias (Arias, et ál, 2018) construyó unos lineamientos de política que se proponen para fortalecer los programas de formación de educadores y atender las problemáticas que las comunidades educativas identifican en torno a las desigualdades en la calidad.

Esta propuesta se realizó, entre otras fuentes, a partir de la activa participación de profesores universitarios de las licenciaturas del país en foros locales y nacionales, ${ }^{19}$ en el estudio de las políticas educativas de las

18 Los modelos de práctica deben contener: 1) justificación e importancia de la práctica pedagógica, 2) líneas de trabajo, 3) enfoque pedagógico, 4) tipos de modalidad de práctica, 5) formas de monitoreo y sistematización, 6) formas y enfoque de evaluación (MEN, 2016).

19 En el marco de la investigación, además de la exploración documental y normativa, hubo interés en identificar la perspectiva y los análisis que hacen profesores y directivos vinculados a las licenciaturas. En tal sentido, se realizaron doce foros regionales en las ciudades de Bogotá, Medellín, Quibdó, Popayán, Barranquilla, Montería, Riohacha, Pereira, Floridablanca, Tunja y Florencia, en los que participaron representantes del 52 IES públicas y privadas que desarrollaban algún programa de licenciatura. Además, se hicieron dieciocho visitas in situ en las que participaron 104 profesores responsables de los procesos de autoevaluación y acreditación, y, en algunos casos, se contó con directivos y estudiantes. 
últimas décadas y en la caracterización de la situación de los programas de licenciatura, a partir de la noción de justicia social que postula Nancy Fraser (2008). Para la autora, en las condiciones actuales del capitalismo, la real participación de los ciudadanos en el marco de las construcciones políticas solo es posible cuando se combinan acertadamente acciones de redistribución y de reconocimiento. Para el caso de las políticas de formación de docentes, este planteamiento se traduce en que el Estado atienda las condiciones materiales, de infraestructura y financieras que se constituyen en barreras para la cualificación de los programas de licenciatura, así como las cuestiones que atañen a la identidad, el prestigio social y el reconocimiento del saber docente como interlocutor válido en el momento de la formulación de políticas educativas, en paridad de condiciones respecto a los funcionarios gubernamentales que las expiden. Para Fraser (2008), "superar la injusticia significa desmantelar los obstáculos institucionalizados que impiden a algunos participar a la par con otros, como socios con pleno derecho en la interacción social" (p. 39).

La propuesta, por tanto, nace de una concepción de educación alternativa a la asumida por parte de los gobiernos de las últimas décadas, en el sentido de que toma distancia de la idea de calidad asociada a la noción de capital humano que ha estructurado los planes nacionales de desarrollo, por lo menos desde 1998 en adelante, en el entendido de que la supuesta mejora de la calidad de la educación que favorece la acreditación conduce a formar y promover el desarrollo de un capital humano altamente calificado que responda a las necesidades económicas, sociales y productivas del país. Los maestros en este proceso se constituyen en la principal herramienta para el desarrollo efectivo de las competencias y aprendizajes de los estudiantes. El sofisma del capital humano, inspirado en los organismos multilaterales, ha estructurado las políticas educativas de las últimas décadas que afectan sustancialmente la dinámica de los programas de licenciatura, en procura de estándares internacionales y que, bajo el discurso de la eficiencia, la calidad y la medición ha cooptado negativamente el concepto de evaluación desde unos parámetros ajenos a la lógica educativa.

Contra aquella idea que concibe la educación como mercado y las IES como empresas que deben competir para reclutar los mejores bachilleres (Ser Pilo Paga, Ser Pilo Paga Profe, Generación E, etc.), la presente propuesta reivindica la educación como un bien público, a las universidades como nichos autónomos de pensamiento, y a la calidad como la posibilidad colectiva de pensar el propio horizonte y de actuar sobre aquellos aspectos que podrían mejorar el proyecto académico escogido. En este escenario, 
de los actores de la educación se relaciona con la naturaleza del compromiso académico y formativo que asuma la universidad, y con la máxima realización posible de ese proyecto en las condiciones cambiantes de su puesta en práctica, atendiendo a las exigencias propias del campo de conocimiento y al ethos académico y formativo propio de la educación superior. (Arias et ál., 2018, p. 356).

Además, el centro de la presente propuesta reconoce la diversidad cultural en la que las IES ejercen su labor, y la variedad territorial que no solo definen matices en las prácticas y procesos formativos, y marca particularidades en las necesidades de los docentes en formación, sino que también entiende que las injustas condiciones sociales y políticas en las que las IES ejercen su función definen la desigualdad en las manifestaciones de la calidad. De manera que la responsabilidad de cualificar los programas no recae exclusivamente sobre el resorte de sí mismos, sino que compete al Estado y a sus instituciones garantizar las condiciones para que la cualificación permanente sea posible.

En esta dirección, se formularon una serie de desplazamientos que buscan transformar la perspectiva u orientación que se ha privilegiado y promover visiones alternativas en las que se enmarcarían nuevas estrategias de política inspiradas en otras maneras de entender la calidad y los cambios en la formación de los maestros. A continuación, se presentan los aportes que configuran los principios organizadores de la acción y el cambio desde una perspectiva educativa y que pueden resultar pertinentes para diversas comunidades interesadas en este debate. ${ }^{20}$

\section{Del Estado evaluador a un Estado que garantiza la realización de los derechos y aporta en la cualificación de la educación}

A partir del reconocimiento de la educación como un derecho, un bien público y común, se reclama un Estado que no solo garantice el acceso y la permanencia de los educandos en todos sus niveles y para todos los sectores sociales, sino que también promueva procesos de cualificación de la educación. Esto implica no reducir el papel del Estado a formular marcos de acción, supervisar e informar y consolidar indicadores orientados a establecer comparaciones y formas de competitividad entre los actores y agentes de la educación superior, pues esto disminuye su potencialidad como Estado garante de derechos y minimiza las posibilidades de transformación de la educación y de ampliación de su compromiso con el mejoramiento de las condiciones de vida de todos.

20 La propuesta se encuentra especificada en detalle en el capítulo cinco del libro derivado del informe final de la investigación de Arias et ál. (2018) que da lugar al presente artículo. 


\section{De la regulación y el control sobre la profesión docente a su reconocimiento y valoración social}

Si se asume que los educadores cumplen la función esencial de formar las nuevas generaciones como herederas de la ciencia, el conocimiento, los saberes y de los demás bienes y valores de la cultura que requiere la vida ciudadana, es necesario redimensionar la valoración social de la profesión docente. Esta tarea demanda afectar factores culturales, sociales, políticos y económicos que han generado y mantenido condiciones de subvaloración de la profesión docente, y que han privilegiado formas de intervención asociadas a la evaluación de su eficacia desde miradas reduccionistas.

\section{De la meritocracia y la exclusión a la democratización en el acceso a mejor educación para todos los educadores}

Es necesario entender que las diferencias de calidad en la formación de los educadores están asociadas a condiciones de desigualdad en la distribución de los recursos, en las formas de reconocimiento y valoración de la experiencia, en las condiciones socioculturales y económicas de los sujetos y en las modalidades de participación de las comunidades educativas y académicas, y en la definición de las políticas que los afectan. Solo si se avanza en la transformación de estas condiciones, y no simplemente desde la selección de los mejores docentes, la generación de incentivos para algunos o el impulso de la competencia entre programas, se hace posible una mejor educación para todos.

\section{De la consulta y validación en la construcción de la política pública a la participación amplia y activa de las comunidades educativas}

La paridad en la participación de quienes estructuran y llevan a cabo los programas de formación de educadores en el país implica la superación de los obstáculos institucionales que impiden la participación de las comunidades académicas de la educación en la formulación y desarrollo de las políticas que transforman el sentido y las formas de reconocimiento de su práctica. Por tanto, no basta consultar a los educadores o a las instituciones para validar procesos de reforma, se requiere asegurar su participación en las discusiones, definiciones e implementaciones de las políticas educativas. 


\section{De la respuesta pragmática e instrumental frente a la evaluación externa a una evaluación como comprensión y reelaboración de la experiencia}

La evaluación, entendida como reflexión sobre la experiencia de los programas de formación de maestros, sobre su potencial transformador y sobre su propia dinámica de mejoramiento permanente, hace posible la articulación de los proyectos educativos y las visiones de futuro recogidas en ellos con sus contextos socioculturales. Este tipo de articulación favorece el mejoramiento cualitativo de los programas, la transformación de esos contextos y asegura una formación más coherente con la tarea cultural de los educadores. La pragmática de la evaluación que se expresa en las prácticas que dan respuesta a las exigencias del sistema de aseguramiento de la calidad es apenas un nivel de todas las potencialidades que ofrece el reconocimiento de la experiencia y de sus posibilidades de cualificación.

\section{De la competitividad y el aislamiento a la solidaridad y la cooperación entre programas y comunidades centradas en la formación de maestros}

Articular esfuerzos para fortalecer la formación inicial de los maestros implica establecer relaciones de solidaridad y cooperación que hagan posible que las universidades y los programas con mayores desarrollos apoyen integralmente a las instituciones que presenten dificultades en la identificación y materialización de sus potencialidades de transformación. Este referente ético de acción obliga a cambiar la mirada a las alianzas que ya no rentarán para indicadores individuales de cada universidad o programa, sino que permitirán una cualificación sustancial del sistema de formación de los educadores y en general de la profesión docente y de la educación.

Los principios antes formulados se traducen en unos lineamientos que se estructuran sobre varios ejes, cada uno de los cuales despliega un conjunto de estrategias que pretenden responder a los problemas identificados y que implican, a su vez, tres distintos ámbitos de responsabilidad (Estado, IES y programas de formación), porque el asunto de la calidad no puede ser responsabilidad exclusiva de los programas, tal como hasta ahora se ha exigido. En el presente escrito se retoman y sintetizan dos de sus ejes centrales.

Respecto al primer eje, potenciar la formación de educadores, se espera que los organismos estatales (MEN, CNA, Minciencias, Secretarías de Educación, comités de capacitación docente) promuevan políticas que articulen la formación de licenciados con la formación en ejercicio y la 
posgradual, con la participación real de las comunidades académicas; que estos entes generen espacios para la formulación y el seguimiento de las políticas de formación de licenciados, atendiendo las especificidades regionales y el conocimiento acumulado en las facultades de educación y en las normales.

Al Estado le compete mejorar las condiciones laborales de los docentes, gestionar y financiar programas de formación y dinamizar los Comités Territoriales de Capacitación Docente para la cualificación y articulación de la formación inicial y en servicio; también, aumentar el presupuesto para mejorar las condiciones académicas y de infraestructura que favorezcan la calidad de los programas en los ámbitos regional y nacional, generar una distribución presupuestal diferenciada, considerando las necesidades específicas de los territorios donde están las IES públicas, así como atender la histórica situación de exclusión de las poblaciones discriminadas y marginadas. Asimismo, realizar estudios que ponderen los efectos de la vinculación de profesionales no licenciados a la educación básica y media, sobre las necesidades de la formación en ejercicio y formular políticas en correspondencia con estos resultados. La política educativa debe dejar de modificarse dependiendo de la agenda económica de los organismos multilaterales y formularse, más bien, sobre la base de estudios, diagnósticos y la participación activa y cualificada de las comunidades académicas.

Respecto a este mismo eje, en el ámbito de responsabilidad de las instituciones formadoras y sus asociaciones, se plantea la necesidad de articular la formación de educadores y la investigación tanto en la formación inicial, como en servicio y posgradual; desarrollar estudios interinstitucionales sobre problemáticas que afectan la calidad, a partir de alianzas con entes territoriales, universidades y normales enfocados en la investigación y en el fortalecimiento de esta formación con agendas a mediano y largo plazo; buscar el diálogo y la colaboración permanente entre profesores de la universidad y de la Educación Básica en búsqueda de sinergias y de producción de saber pedagógico. Además, las mismas instituciones formadoras y sus asociaciones pueden incentivar escenarios de comunicación respecto a la importancia de la labor social y política de los educadores, participar decididamente en la formulación de políticas y trazar orientaciones institucionales direccionadas a reducir las desigualdades de las trayectorias educativas de los estudiantes de licenciatura, su deserción y no graduación; también crear espacios interinstitucionales de intercambio, de investigación y cualificación de los programas de formación docente.

En esta misma dirección, los programas han de contribuir con la formulación de políticas educativas a partir de la sistematización de sus experiencias, con investigaciones referidas a la formación inicial, a las transformaciones curriculares, y con la generación de espacios de socialización y debate 
con docentes simultáneamente en ejercicio y en formación. Las IES y los programas de licenciatura deben exigir mayor responsabilidad del Estado para poder ofrecer una educación en las mejores condiciones, y a la vez rechazar ser objeto de intervención y control por parte del MEN, y asumir su autonomía para proponer iniciativas y políticas acordes con su misión y su contexto.

Otro eje de la propuesta por desarrollar consiste en redimensionar el Sistema de Aseguramiento de la Calidad, lo que también comporta diferentes niveles de responsabilidad. En el caso del Estado y las instituciones encargadas de dirigir la Educación Superior se deben evaluar los casi veinte años de funcionamiento del sistema, con sus logros y deficiencias y hacer públicos los resultados; hay que armonizar los procesos de registro calificado y los de acreditación en una perspectiva sistémica, evitando dobles tareas y desgaste. Asimismo se deben cualificar permanentemente los pares evaluadores del CNA y aprovechar su saber para no empezar cada tanto de cero; depurar los referentes, criterios e indicadores de calidad en los documentos oficiales, en atención a la simplificación de temas, unificación de esfuerzos, y sobre todo contemplar el reconocimiento de la diversidad regional e impacto de los programas de licenciatura en los contextos donde se encarnan.

Igualmente, se propone fomentar las alianzas y la cooperación entre instituciones y programas, así como diversificar los productos y procesos de la investigación educativa de acuerdo con su naturaleza. El Sistema de Aseguramiento de Calidad, primero que todo debe ser eso: un sistema, que articula las partes con el todo, que centraliza información y que sirve de apoyo a las labores de las IEs y los programas, y no, como hasta ahora, pensado para dar respuesta a matrices adustas, gestadas desde el centro del país, que poco tienen que ver con las necesidades y requerimientos de las regiones y las comunidades académicas.

Sobre este mismo redimensionamiento, las IES formadoras de licenciados y sus agremiaciones tienen el compromiso de promover el trabajo colaborativo entre programas e instituciones, con miras a solucionar problemáticas comunes; construir un observatorio pedagógico regional de programas de formación inicial, que proponga políticas que detecten vacíos, que identifiquen fortalezas y que atiendan los contextos donde los programas funcionan; además, las IES deben jalonar la formación de una mesa intersectorial con la presencia del Estado para que se propenda por la investigación y la mejora de la calidad de la educación de las regiones desde una instancia superior.

Finalmente, los programas de formación y sus comunidades académicas pueden redimensionar el Sistema de Aseguramiento de la Calidad al fomentar una autoevaluación reflexiva y participativa orientada a mejorar sus carreras 
desde la perspectiva del ethos institucional y la identidad académica de los propios programas, así como desde el reconocimiento de la especificidad regional. Las mismas carreras pueden emprender evaluaciones ligadas a la investigación, entendida como una transformación social, de la propia universidad y del programa, así como cualificar a los equipos que dirigen la evaluación a partir de los aprendizajes previos y los caminos recorridos. En síntesis, los programas de licenciatura, las normales y las IES que tienen programas de formación de licenciados deben ser el centro del sistema, no solo como destinatarios de las políticas educativas en procura de la calidad, sino como parte de este, para dinamizarlo, analizarlo y proponer acciones de mejora de acuerdo con sus propias opciones y necesidades.

\section{Reflexiones finales}

Como se mostró al analizar los efectos de la acreditación obligatoria de las licenciaturas, las políticas de calidad basadas en las teorías del capital humano han incentivado la desigualdad porque han privilegiado la competitividad y la medición del déficit a partir de la identificación de unos referentes denominados indicadores, que se deben alcanzar por efecto del esfuerzo individual, en este caso de los programas de formación. Como lo señala Sebastián Plá, su objetivo "no es producir la igualdad, sino por el contrario, crear dispositivos que con base en la equidad y la inclusión produzcan desigualdades legítimas" (2018, p. 19).

En este artículo proponemos transitar de las teorías del capital humano que se han privilegiado como marco de análisis y como referente técnico para la formulación de las políticas educativas, a una comprensión de la calidad derivada de mecanismos efectivos de distribución de recursos para los programas de licenciatura, de la participación efectiva en las decisiones políticas del sector educativo y del reconocimiento social de la profesión docente, lo cual incluye mejoras significativas en su estatus y en su condición salarial.

\section{Referencias}

Arias, D., Díaz, O., Garzón, I., León, A., Rodríguez, S. y Valbuena, E. (2018). Entre las exigencias de calidad y las condiciones de desigualdad: Formación inicial de profesores en Colombia. Universidad Pedagógica Nacional y Colciencias. https://www.academia.edu/36808796/Entre_ las_exigencias_de_calidad_y_las_condiciones_de_desigualdad_Formaci\%C3\%B3n_inicial_de_profesores_en_Colombia

Atehortúa, A. (2017, 27 de abril). En defensa de las licenciaturas. El Espectador. https://www.elespectador.com/opinion/en-defensa-de-las-licenciaturas-columna-691382 
Bruns, B. y Luque, J. (2014). Profesores excelentes. Cómo mejorar el aprendizaje en América Latina y el Caribe. Banco Mundial. https://openknowledge.worldbank.org/bitstream/handle/10986/20488/Great\%20 Teachers_Spanish.pdf? sequence $=8 \&$ isAllowed $=y$ Congreso de la República de Colombia. (2015, 9 de junio). Ley 1753, por la cual se expide el Plan Nacional de Desarrollo 2014-2018 Todos por un Nuevo País. https://colaboracion.dnp.gov.co/CDT/Normograma/ Ley\%201753\%20de\%202015.pdf

Departamento Nacional de Planeación (DNP). (2015). Plan Nacional de Desarrollo 2014-2018. Todos por un nuevo país. Imprenta Nacional de Colombia. https://colaboracion.dnp.gov.co/CDT/PND/PND\%20 2014-2018\%20Tomo\%201\%20internet.pdf

Departamento Nacional de Planeación (DNP). (2018). Plan Nacional de Desarrollo 2018-2022 Pacto por Colombia, Pacto por la Equidad. https:// www.dnp.gov.co/DNPN/Paginas/Plan-Nacional-de-Desarrollo.aspx

Fraser, N. (2008). Escalas de justicia. Herder.

García, S., Maldonado, D., Perry, G., Rodríguez, C. y Saavedra, J. E. (2014). Tras la excelencia docente. Cómo mejorar la calidad de la educación para todos los colombianos. Fundación Compartir. http://www.fundacioncompartir.org/pdf/Tras\%20la\%20excelencia\%20docente\%20 -\%20estudio\%20final.pdf

Ministerio de Educación Nacional. (2015, 17 de noviembre). Circular 61. Precisiones sobre los procesos de registro calificado de programas acreditados. http://186.154.251.228:85/docs/pdf/circular_mineducacion_0061_2015.pdf

Ministerio de Educación Nacional. (2015, 17 de diciembre). Decreto 2450, por el cual se reglamentan las condiciones de calidad para el otorgamiento y renovación del registro calificado de los programas académicos de licenciatura y los enfocados a la educación, y se adiciona el Decreto 1075 del 2015, único reglamentario del sector educación. https:// www.mineducacion.gov.co/1759/articles-357048_recurso_1.pdf

Ministerio de Educación Nacional. (2016, 3 de febrero). Resolución 2041, por la cual se establecen las características específicas de calidad de los programas de licenciatura para la obtención, renovación o modificación del registro calificado. Imprenta Nacional. https:// www.mineducacion.gov.co/1759/articles-356982_recurso_1.pdf

Ministerio de Educación Nacional. (2016, 16 de febrero). Circular 14. Precisiones sobre el registro calificado y la acreditación de las licenciaturas. https://www.mineducacion.gov.co/1759/articles-357355_ recurso_1.pdf

Ministerio de Educación Nacional. (2016, 16 de junio). Directiva 30. Invitación a la realización de convenios entre instituciones de educación superior (IES) y las entidades territoriales certificadas en educación (ETC) 
para que los estudiantes de los programas de licenciatura puedan desarrollar su práctica pedagógica en establecimientos educativos oficiales. https://www.mineducacion.gov.co/1759/articles-357401_ recurso_normatividad.pdf

Ministerio de Educación Nacional. (2017, 28 de febrero). Circular 16. Calendario de visitas de evaluación externa. https://www.mineducacion.gov.co/1759/articles-360188_recurso_normatividad.pdf

Ministerio de Educación Nacional. (2017, 28 de mayo). Decreto 892, por el cual se crea un régimen transitorio para la acreditación en alta calidad de los programas académicos de licenciaturas a nivel de pregrado que son ofrecidos en departamentos donde se localizan municipios priorizados para la implementación de los Programas de Desarrollo con Enfoque Territorial (PDET). http://es.presidencia.gov. co/normativa/normativa/DECRETO\%20892\%20DEL\%2028\%20DE\%20 MAYO\%20DE\%202017.pdf

Ministerio de Educación Nacional. (2017, 15 de septiembre). Resolución 18583, por la cual se ajustan las características específicas de calidad de los programas de licenciatura para la obtención, renovación o modificación del registro calificado, y se deroga la Resolución 2041 del 2016. https://www.usbcali.edu.co/sites/default/files/resolucion_final_18583_de_2017deroga_2041.pdf

Ministerio de Educación Nacional. (2018, 20 de abril). Circular 18. Información sobre la propuesta de modificación del Decreto 1075 del 2015 en lo referente al sistema de aseguramiento de la calidad de la educación superior. https://www.mineducacion.gov.co/1759/articles-369022_recurso_1.pdf

Ministerio de Educación Nacional. (2018, 25 de julio). Decreto 1280, por el cual se reglamenta el sistema de aseguramiento de la calidad de la educación superior, el registro calificado de que trata la Ley 1188 del 2008 y los artículos 53 y 54 de la Ley 30 de 1992 sobre acreditación, por lo que se subrogan los capítulos 2 y 7 del título 3 de la parte 5 del libro 2 del Decreto 1075 del 2015-único reglamentario del sector educación. http://es.presidencia.gov.co/normativa/normativa/DECRETO\%201280\%20DEL\%2025\%20DE\%20JULIO\%20DE\%202018.pdf

Ministerio de Educación Nacional. (2018, 24 de diciembre). Decreto 2389, por el cual se modifica el artículo 3 del Decreto 1280 del 2018 "por el cual se reglamenta el sistema de aseguramiento de la calidad de la educación superior, el registro calificado de que trata la ley 1188 del 2008 y los artículos 53 y 54 de la ley 30 de 1992 sobre acreditación, por lo que se subrogan los capítulos 2 y 7 del título 3 de la parte 5 del libro 2 del Decreto 1075 del 2015-único reglamentario del sector educación". https://www.mineducacion.gov.co/1759/articles-380231_archivo_pdf.pdf 
Ministerio de Educación Nacional. (2019, 25 de julio). Decreto 1330, por el cual se sustituye el capítulo 2 y se suprime el capítulo 7 del título 3 de la parte 5 del libro 2 del Decreto 1075 del 2015-único reglamentario del sector educación. Diario Oficial 51.025. https:// www.mineducacion.gov.co/1759/articles-387348_archivo_pdf.pdf

Plá, S. (2018). Calidad educativa. Historia de una política para la desigualdad. Universidad Autónoma de México, Instituto de Investigaciones sobre la Universidad y la Educación.

Urrutia, M. y Robles, C. (2019). Mercado y gasto social: la agenda pendiente para la equidad en Colombia. Desarrollo y Sociedad, 82, 73-101. 